\title{
Management of patients with prostate cancer: evolution towards a risk-stratified approach
}

Growing evidence exists that patients with localized prostate cancer have substantial differences in their management needs, depending on their individual risks of adverse outcomes. Now, results of an extensive analysis have confirmed that this evidence is beginning to be adopted into clinical practice: an analysis of the Cancer of the Prostate Strategic Urologic Research Endeavour (CAPSURE) database reveals a trend towards increased surveillance of patients with low-risk localized prostate cancer, combined with a greater incidence of curative treatment of patients with high-risk localized prostate cancer between 1990 and 2013.

The CAPSURE database is a US national registry containing information on $>10,000$ men diagnosed with prostate cancer at one of 45 centres. In this study, patients were assigned a specific risk category based on individual Cancer of the Prostate Risk Assessment (CAPRA) scores, followed by an analysis of treatment trends over 5 yearly intervals. Use of active surveillance, even in patients with low-risk disease, remained low (6.7$14.3 \%$ of patients) for the majority of the study duration, with a substantial increase after 2010 .

\section{4 ...screening for prostate} cancer should be considered in this population... 77

Among patients with intermediate-risk, or high-risk prostate cancer, a notable trend was observed towards decreased use of androgen-deprivation therapy (ADT) in 2013, compared with earlier time points. A trend towards increased use of radical prostatectomy, reflecting a move towards the use of therapies that are more likely to be potentially curative was also reported in this patient group.
In a subgroup of men $\geq 75$ years of age, a substantial increase in use of watchful waiting/active surveillance was, again, observed in patients with low-risk disease. Radiation or ADT remained the mainstays of treatment for patients in this subgroup, reflecting the risks of adverse outcomes of surgery in this patient group.

These findings indicate that the clinical management of patients with prostate cancer is evolving rapidly as new evidence becomes available. The authors speculate that screening for prostate cancer should be considered in this population: a risk of overtreatment of patients with low-risk disease was previously considered a major reason not to screen for prostate cancer.

Peter Sidaway

Original article Cooperberg, M. R. \& Carroll, P. R. Trends in management for patients with localized prostate cancer 1990-2013. JAMA 314, 80-82 (2015) 